\title{
Orlicz spaces which are AM-spaces
}

\author{
By \\ C. E. Finol, H. HudziK*) and L. Maligranda**)
}

\begin{abstract}
The Orlicz function and sequence spaces which are AM-spaces are characterized for both the Luxemburg-Nakano and the Amemiya (Orlicz) norm.
\end{abstract}

1. Preliminaries. Let $(\Omega, \Sigma, \mu)$ be a positive complete $\sigma$-finite measure space and $L^{0}=L^{0}(\mu)$ the space of all (equivalence classes of) $\Sigma$-measurable real functions on $\Omega$.

Consider an Orlicz function $\varphi:[0, \infty) \rightarrow[0, \infty]$, i.e., a convex nondecreasing function vanishing at zero (not identically 0 or $\infty$ on $(0, \infty)$ ) and define the functional $I_{\varphi}: L^{0}(\mu) \rightarrow[0, \infty]$ by the formula

$$
I_{\varphi}(x)=\int_{\Omega} \varphi(|x(t)|) d \mu .
$$

The Orlicz space $L_{\varphi}(\mu)$ is defined by $L_{\varphi}(\mu)=\left\{x \in L^{0}(\mu): I_{\varphi}(x / \lambda)<\infty\right.$ for some $\left.\lambda>0\right\}$. This space is a Banach space with the following three norms: the Luxemburg-Nakano norm

$$
\|x\|_{\varphi}=\inf \left\{\lambda>0: I_{\varphi}(x / \lambda) \leqq 1\right\}
$$

the Amemiya norm

$$
\|x\|_{\varphi}^{A}=\inf _{k>0} \frac{1}{k}\left(1+I_{\varphi}(k x)\right)
$$

and the Orlicz norm

$$
\|x\|_{\varphi}^{0}=\sup \left\{\left|\int_{\Omega} x(t) y(t) d \mu\right|: y \in L_{\varphi^{*}}, I_{\varphi^{*}}(y) \leqq 1\right\},
$$

where the function $\varphi^{*}:[0, \infty) \rightarrow[0, \infty]$ is defined by the formula

$$
\varphi^{*}(u)=\sup \{u v-\varphi(v): v \geqq 0\}
$$

and called complementary to $\varphi$ in the sense of Young (see [5], [8], [9], [10]). For the counting measure $\mu$ on $N$ we obtain the Orlicz sequence space $l_{\varphi}=\left\{x=\left(x_{n}\right)\right.$ : $I_{\varphi}(x / \lambda)=\sum_{n=1}^{\infty} \varphi\left(\left|x_{n}\right| / \lambda\right)<\infty$ for some $\left.\lambda>0\right\}$. It is well known that $\|x\|_{\varphi} \leqq\|x\|_{\varphi}^{0} \leqq 2\|x\|_{\varphi}$

Mathematics Subject Classification (1991): 46E30.

*) Supported by KBN Grant 2 P03A 03110.

**) Supported in part by The Royal Swedish Academy of Sciences grant 9265 (1996). 
and $\|x\|_{\varphi} \leqq 1$ if and only if $I_{\varphi}(x) \leqq 1$ (cf. [5], [8], [9] and [10]). It is also known that $\|x\|_{\varphi}^{0}=\|x\|_{\varphi}^{A}$ for any $x \in L_{\varphi}$ (cf. [4]).

Baron and Hudzik [1] have proved that the only Orlicz spaces $L_{\varphi}$ that are abstract $L_{p}$ spaces $(1<p<\infty)$, i.e. $\|x+y\|_{\varphi}^{p}=\|x\|_{\varphi}^{p}+\|y\|_{\varphi}^{p}$ for $x, y \in L_{\varphi}, x \perp y$, are the Lebesgue spaces $L_{p}$.

In this paper we will consider the limit case, namely we will solve the problem which Orlicz function spaces $L_{\varphi}$ or Orlicz sequence spaces $l_{\varphi}$ are AM-spaces. We will solve this problem for both the Luxemburg-Nakano and the Amemiya (Orlicz) norm. As we will see, Orlicz spaces $L_{\varphi}$ and $l_{\varphi}$ can be AM-spaces iff they are isometric to $L_{\infty}$ or $l_{\infty}$ under the isometry $\lambda$ Id for some $\lambda>0$.

Recall that a subspace $(X,\|\cdot\|)$ of $L^{0}(\mu)$ is said to be a Banach function space if it is a Banach space satisfying the following condition: if $x \in L^{0}, y \in X$ and $|x(t)| \leqq|y(t)| \mu$-a.e., then $x \in X$ and $\|x\| \leqq\|y\|$.

A Banach function space $X=(X,\|\cdot\|)$ is an $A M$-space if

$$
\|\max (x, y)\|=\max (\|x\|,\|y\|) \text { for all } 0 \leqq x, y \in X .
$$

An equivalent and very useful condition on AM-space says that a Banach function space is an AM-space if and only if

$$
\|x+y\|=\max (\|x\|,\|y\|) \text { for all } x, y \in X \text { with } x \perp y,
$$

where $x \perp y$ means that $\mu(\operatorname{supp} x \cap \operatorname{supp} y)=0$ and the support supp $x$ of a function $x \in X$ is defined (up to a set of measure zero) by the formula supp $x=\{t \in \Omega: x(t) \neq 0\}$.

A Banach function space $X$ has the Fatou property if $0 \leqq x_{n} \uparrow x$ with $x_{n} \in X, x \in L^{0}$ and $\sup _{n}\left\|x_{n}\right\|<\infty$ imply $x \in X$ and $\|x\|=\lim _{n \rightarrow \infty}\left\|x_{n}\right\|$ (see [7] and [8]).

The Orlicz space $L_{\varphi}(\mu)$ with both the Luxemburg-Nakano and the Amemiya norm is a Banach function space with the Fatou property.

2. Orlicz spaces over a nonatomic measure space which are AM-spaces. First of all we will prove that if an Orlicz function is finite-valued then the Orlicz function space cannot be an AM-space. We need to define for an Orlicz function $\varphi$ the following two parameters:

$$
u_{0}(\varphi)=\sup \{u \geqq 0: \varphi(u)=0\} \text { and } u_{\infty}(\varphi)=\sup \{u>0: \varphi(u)<\infty\} .
$$

From the definition of Orlicz function we have $u_{0}(\varphi) \leqq u_{\infty}(\varphi), u_{0}(\varphi)<\infty$ and $u_{\infty}(\varphi)>0$.

Theorem 1. (i) If the Orlicz space $L_{\varphi}(\mu)$ with either the Luxemburg-Nakano or the Amemiya norm on a nonatomic measure space $(\Omega, \Sigma, \mu)$ is an AM-space, then $u_{\infty}(\varphi)<\infty$.

(ii) If $u_{\infty}(\varphi)<\infty$, then $L_{\varphi}(\mu) \subset L_{\infty}(\mu)$ and $\|x\|_{\infty} \leqq u_{\infty}(\varphi)\|x\|_{\varphi}$.

Proof. Let $u_{\infty}(\varphi)=\infty$, i.e., let $\varphi$ be a finite-valued function. Take disjoint $A, B \in \Sigma$ and a number $c>u_{0}(\varphi)$ such that $\varphi(c) \mu(A)=1$ and $\varphi(c) \mu(B)=1$. Define

$$
x=c \chi_{A}, \quad y=c \chi_{B} .
$$

Then $I_{\varphi}(x)=\int_{A} \varphi(c) d \mu=\varphi(c) \mu(A)=1$ and so $\|x\|_{\varphi}=1$. Similarly, $\|y\|_{\varphi}=1$ but

$$
I_{\varphi}(x+y)=\int_{A} \varphi(c) d \mu+\int_{B} \varphi(c) d \mu=\varphi(c) \mu(A)+\varphi(c) \mu(B)=2
$$


gives that $\|x+y\|_{\varphi}>1$, and the Orlicz space $L_{\varphi}(\mu)$ with the Luxemburg-Nakano norm $\|\cdot\|_{\varphi}$ does not satisfy (2) which means that it is not an AM-space.

Consider now the Orlicz space with the Amemiya norm and assume that $u_{\infty}(\varphi)=\infty$. For any $c>u_{0}(\varphi)$ there exists $\varepsilon>0$ such that $(1+\varepsilon)\left(u_{0}(\varphi)+\varepsilon\right)<c$. Choose $A \in \Sigma$ such that $0<\mu(A)<\infty$ and $I_{\varphi}\left(c \chi_{A}\right)=\varphi(c) \mu(A) \leqq \varepsilon$. This is possible since our measure is nonatomic. Then

$$
\begin{aligned}
\left\|\chi_{A}\right\|_{\varphi}^{A} & =\inf _{k>0} \frac{1}{k}\left(1+I_{\varphi}\left(k \chi_{A}\right)\right) \leqq \frac{1}{c}\left(1+I_{\varphi}\left(c \chi_{A}\right)\right) \\
& =\frac{1}{c}(1+\varphi(c) \mu(A)) \leqq(1+\varepsilon) / c<1 /\left(u_{0}(\varphi)+\varepsilon\right) .
\end{aligned}
$$

Consider now two cases.

I. There is $k_{0}>0$ such that $\left\|\chi_{A}\right\|_{\varphi}^{A}=\frac{1}{k_{0}}\left(1+I_{\varphi}\left(k_{0} \chi_{A}\right)\right)$.

We will show that $I \varphi\left(k_{0} \chi_{A}\right)>0$. If $u_{0}(\varphi)=0$, then this is obviously true. Let $u_{0}(\varphi)>0$ and assume for the contrary that $I_{\varphi}\left(k_{0} \chi_{A}\right)=0$. Then it must be $k_{0} \leqq u_{0}(\varphi)$, and so $\left\|\chi_{A}\right\|_{\varphi}^{A}=1 / k_{0} \geqq 1 / u_{0}(\varphi)$, a contradiction. Therefore we have indeed $I_{\varphi}\left(k_{0} \chi_{A}\right)>0$. This implies that $0<\varphi\left(k_{0}\right)<\infty$. Let $B \subset A, B \in \Sigma$, be such that $\mu(B)=\mu(A \backslash B)=\mu(A) / 2$. Then

$$
\begin{aligned}
\left\|\chi_{A}\right\|_{\varphi}^{A} & =\left[1+I_{\varphi}\left(k_{0} \chi_{A}\right)\right] / k_{0}>\left[1+I_{\varphi}\left(k_{0} \chi_{B}\right)\right] / k_{0} \geqq \\
& \geqq \inf _{k>0} \frac{1}{k}\left[1+I_{\varphi}\left(k \chi_{B}\right)\right]=\left\|\chi_{B}\right\|_{\varphi}^{A} .
\end{aligned}
$$

We can prove in the same way that

$$
\left\|\chi_{A}\right\|_{\varphi}^{A}>\left\|\chi_{A \backslash B}\right\|_{\varphi}^{A},
$$

and consequently that

$$
\left\|\chi_{B}+\chi_{A \backslash B}\right\|_{\varphi}^{A}=\left\|\chi_{A}\right\|_{\varphi}^{A}>\max \left\{\left\|\chi_{B}\right\|_{\varphi}^{A},\left\|\chi_{A \backslash B}\right\|_{\varphi}^{A}\right\} .
$$

This yields that equality (2) does not hold, which gives that $\left(L_{\varphi}(\mu),\|\cdot\|_{\varphi}^{A}\right)$ is not an AM-space.

II. Assume that $\left\|\chi_{A}\right\|_{\varphi}^{A}<\left[1+I_{\varphi}\left(k \chi_{A}\right)\right] / k$ for any $k>0$. Then

$$
\left\|\chi_{A}\right\|_{\varphi}^{A}=\lim _{k \rightarrow \infty} \frac{1}{k} I_{\varphi}\left(k \chi_{A}\right)=\mu(A) \lim _{k \rightarrow \infty}(\varphi(k) / k) .
$$

Of course case II is possible only when $\left.\lim _{u \rightarrow \infty} \varphi(u) / u\right)<\infty$. Then for $B \subset A, B \in \Sigma$ with $\mu(B)=\mu(A \backslash B)=\mu(A) / 2$, we have

$$
\begin{aligned}
\left\|\chi_{A}\right\|_{\varphi}^{A} & =\mu(A) \lim _{k \rightarrow \infty}(\varphi(k) / k)=2 \mu(B) \lim _{k \rightarrow \infty}(\varphi(k) / k) \\
& \geqq 2 \inf _{k>0} \frac{1}{k}\left[1+I_{\varphi}\left(k \chi_{B}\right)\right]>\inf _{k>0} \frac{1}{k}\left[1+I_{\varphi}\left(k \chi_{B}\right)\right]=\left\|\chi_{B}\right\|_{\varphi}^{A} .
\end{aligned}
$$

In the analogous way we can prove that $\left\|\chi_{A}\right\|_{\varphi}^{A}>\left\|\chi_{A \backslash B}\right\|_{\varphi}^{A}$. Therefore

$$
\left\|\chi_{B}+\chi_{A \backslash B}\right\|_{\varphi}^{A}=\left\|\chi_{A}\right\|_{\varphi}^{A}>\max \left\{\left\|\chi_{B}\right\|_{\varphi}^{A},\left\|\chi_{A \backslash B}\right\|_{\varphi}^{A}\right\} .
$$

This means that $\left(L_{\varphi}(\mu),\|\cdot\|_{\varphi}^{A}\right)$ is not an AM-space, and the proof is complete. 
(ii) (cf. [2]). For $0 \neq x \in L_{\varphi}(\mu)$ let $A=\left\{t \in \Omega:|x(t)|>u_{\infty}(\varphi)\|x\|_{\varphi}\right\}$. Since $\varphi\left(x \chi_{A} /\|x\|_{\varphi}\right)=\infty$ it follows that $\infty \cdot \mu(A)=I_{\varphi}\left(x \chi_{A} /\|x\|_{\varphi}\right) \leqq I_{\varphi}\left(x /\|x\|_{\varphi}\right) \leqq 1$. This gives that $\mu(A)=0$, i.e.,

$$
|x(t)| \leqq u_{\infty}(\varphi)\|x\|_{\varphi} \quad \mu \text {-a.e. on } \Omega,
$$

and (ii) follows.

Theorem 2. Let $(\Omega, \Sigma, \mu)$ be a nonatomic measure space. The following assertions are equivalent:

(i) An Orlicz space $\left.L_{\varphi}(\mu)\right)$ with the Luxemburg-Nakano norm is an AM-space.

(ii) $u_{\infty}(\varphi)<\infty$ and $\varphi\left(u_{\infty}(\varphi)\right)=0$ if $\mu(\Omega)=\infty$ or $\varphi\left(u_{\infty}(\varphi)\right) \mu(\Omega) \leqq 1$ if $\mu(\Omega)<\infty$.

(iii) $L_{\varphi}(\mu)=L_{\infty}(\mu)$ and there is a constant $k>0$ such that $\|x\|_{\varphi}=k\|x\|_{\infty}$ for every $x \in L_{\varphi}(\mu)$.

Proof. (i) $\Rightarrow$ (ii). The fact that $u_{\infty}(\varphi)<\infty$ follows from Theorem 1. Assume then that $\varphi\left(u_{\infty}(\varphi)\right) \mu(\Omega)>1$, where $0 \cdot \infty=0$ by definition. Then $\varphi\left(u_{\infty}(\varphi)\right)>0$ must hold. Take $A, B \subset \Omega, A, B \in \Sigma$ such that $A \cap B=\emptyset, \varphi\left(u_{\infty}(\varphi)\right) \mu(A)=1$ and $0<\varphi\left(u_{\infty}(\varphi)\right) \mu(B) \leqq 1$.

Define

$$
x=u_{\infty}(\varphi) \chi_{A}, \quad y=u_{\infty}(\varphi) \chi_{B} .
$$

Since $I_{\varphi}(x)=1$, we get directly $\|x\|_{\varphi}=1$. The conditions $I_{\varphi}(y) \leqq 1$ and $I_{\varphi}(y / \lambda)=\infty$ for any $\lambda \in(0,1)$ imply that $\|y\|_{\varphi}=1$. However, $I_{\varphi}(x+y)>1$, whence it follows that $\|x+y\|_{\varphi}>1$, i.e.

$$
\|x+y\|_{\varphi}>\max \left\{\|x\|_{\varphi},\|y\|_{\varphi}\right\}
$$

and (i) does not hold. This finishes the proof of the implication.

(ii) $\Rightarrow$ (iii). We will prove first that (ii) implies that $L_{\varphi}(\mu)=L_{\infty}(\mu)$. Assume that $x \in L_{\infty}(\mu)$. Then, by (ii),

$$
I_{\varphi}\left(u_{\infty}(\varphi) x /\|x\|_{\infty}\right) \leqq 1 \text {, i.e. } x \in L_{\varphi}(\mu)
$$

and

$$
\|x\|_{\varphi} \leqq u_{\infty}(\varphi)^{-1}\|x\|_{\infty} .
$$

Assume now that $x \in L_{\varphi}(\mu)$, i.e., there is $\lambda>0$ such that $d=I_{\varphi}(\lambda x)<\infty$. Then by the convexity of $I_{\varphi}$, we obtain

$$
I_{\varphi}(\lambda x / \max \{1, d\}) \leqq I_{\varphi}(\lambda x) / \max \{1, d\} \leqq 1 .
$$

This means that

$$
\lambda|x(t)| / \max \{1, d\} \leqq u_{\infty}(\varphi) \quad \mu \text {-a.e. in } \Omega,
$$

i.e. $x \in L_{\infty}(\mu)$. Since

$$
I_{\varphi}\left(u_{\infty}(\varphi) x /\left(\lambda\|x\|_{\infty}\right)\right)=\infty \quad \forall \lambda \in(0,1),
$$

we get

$$
\|x\|_{\varphi} \geqq u_{\infty}(\varphi)^{-1}\|x\|_{\infty} .
$$


Since the opposite inequality was also proved we obtain the equality

$$
\|x\|_{\varphi}=u_{\infty}(\varphi)^{-1}\|x\|_{\infty} \quad \forall x \in L_{\varphi}(\mu) .
$$

The implication (iii) $\Rightarrow$ (i) follows immediately from the fact that $L_{\infty}(\mu)$ is an AM-space. This finishes the proof of the theorem.

The next result concerns Orlicz spaces with the Amemiya norm.

Theorem 3. Let $(\Omega, \Sigma, \mu)$ be a nonatomic measure space. Then an Orlicz space $L_{\varphi}(\mu)$ with the Amemiya norm is an AM-space if and only if

$$
u_{0}(\varphi)>0, u_{\infty}(\varphi)<\infty \quad \text { and } \quad u_{0}(\varphi)=u_{\infty}(\varphi)
$$

Proof. Sufficiency. Assume that $\varphi$ satisfies condition (4), i.e.,

$$
\varphi(u)= \begin{cases}0 & \text { for } 0 \leqq u \leqq u_{0}, \\ \infty & \text { for } u>u_{0},\end{cases}
$$

for some $u_{0}>0$. Then $L_{\varphi}(\mu)=L_{\infty}(\mu),\|x\|_{\varphi}=u_{0}^{-1}\|x\|_{\infty}$ for every $x \in L_{\varphi}(\mu)$ and

$$
\begin{aligned}
\|x\|_{\varphi}^{A} & =\inf _{k>0, I_{\varphi}(k x)<\infty} \frac{1}{k}\left(1+I_{\varphi}(k x)\right)=\inf \left\{1 / k: k>0 \text { and } I_{\varphi}(k x)<\infty\right\} \\
& =\inf \left\{1 / k: k>0 \text { and } k|x(t)| \leqq u_{0} \mu \text {-a.e. in } \Omega\right\}=u_{0}^{-1}\|x\|_{\infty} .
\end{aligned}
$$

It is obvious that the last equality implies that $\left(L_{\varphi}(\mu),\|\cdot\|_{\varphi}^{A}\right)$ is an AM-space.

Necessity. From Theorem 1 we have that $u_{\infty}(\varphi)<\infty$. If $u_{0}(\varphi)=0$, then the Amemiya norm $\|\cdot\|_{\varphi}^{A}$ is strictly monotone, i.e., $0 \leqq x \leqq y, x \neq y \mu$-a.e. imply $\|x\|_{\varphi}^{A}<\|y\|_{\varphi}^{A}$ (see [3]), so $\|\cdot\|_{\varphi}^{A}$ does not satisfy condition (2). For the sake of completeness, we will repeat here the proof of strict monotonicity of $\|\cdot\|_{\varphi}^{A}$ from [3]. The assumption $\lim _{u \rightarrow \infty} \varphi(u) / u=\infty$, which follows by $u_{\infty}(\varphi)<\infty$, gives that $\|y\|_{\varphi}^{A}=\left[1+I_{\varphi}\left(k_{0} y\right)\right] / k_{0}$ for some positive $k_{0}$ (cf. [10]). Then, since the convex function $\varphi$ is superadditive (cf. [5], 1.19),

$$
I_{\varphi}\left(k_{0} y\right)=I_{\varphi}\left(k_{0}(y-x)+k_{0} x\right) \geqq I_{\varphi}\left(k_{0}(y-x)\right)+I_{\varphi}\left(k_{0} x\right)
$$

and so

$$
\begin{aligned}
\|y\|_{\varphi}^{A} & =\left[1+I_{\varphi}\left(k_{0} y\right)\right] / k_{0} \geqq\left[1+I_{\varphi}\left(k_{0}(y-x)\right)+I_{\varphi}\left(k_{0} x\right)\right] / k_{0} \\
& =\left[1+I_{\varphi}\left(k_{0} x\right)\right] / k_{0}+I_{\varphi}\left(k_{0}(y-x)\right) / k_{0} \\
& \geqq\|x\|_{\varphi}^{A}+I_{\varphi}\left(k_{0}(y-x)\right) / k_{0}>\|x\|_{\varphi}^{A} .
\end{aligned}
$$

The last strict inequality follows from the facts that $u_{0}(\varphi)=0$ (or equivalently $\varphi(u)>0$ for $u>0$ ) and $x \neq y$.

Assume now that $u_{0}(\varphi)>0$ and $u_{0}(\varphi)<u_{\infty}(\varphi)$. Take $\varepsilon>0$ such that $(1+\varepsilon) u_{0}(\varphi)<u_{\infty}(\varphi)-\varepsilon$ and choose $A \in \Sigma$ with $0<\mu(A)<\infty$ and such that

$$
I_{\varphi}\left(\left(u_{\infty}(\varphi)-\varepsilon\right) \chi_{A}\right)=\varphi\left(u_{\infty}(\Phi)-\varepsilon\right) \mu(A) \leqq \varepsilon .
$$

Then

$$
\begin{aligned}
\left\|\chi_{A}\right\|_{\varphi}^{A} & =\inf _{k>0} \frac{1}{k}\left(1+I_{\varphi}\left(k \chi_{A}\right)\right) \\
& \leqq\left[1+I_{\varphi}\left(\left(u_{\infty}(\varphi)-\varepsilon\right) \chi_{A}\right)\right] /\left(u_{\infty}(\varphi)-\varepsilon\right) \\
& =\left[1+\varphi\left(u_{\infty}(\varphi)-\varepsilon\right) \mu(A)\right] /\left(u_{\infty}(\varphi)-\varepsilon\right) \leqq(1+\varepsilon) /\left(u_{\infty}(\varphi)-\varepsilon\right) \\
& <1 / u_{0}(\varphi)
\end{aligned}
$$


and, similarly as in the proof of Theorem 1 ,

$$
\left\|\chi_{B}+\chi_{A \backslash B}\right\|_{\varphi}^{A}=\left\|\chi_{A}\right\|_{\varphi}^{A}>\max \left\{\left\|\chi_{B}\right\|_{\varphi}^{A},\left\|\chi_{A \backslash B}\right\|_{\varphi}^{A}\right\},
$$

where $B \subset A, B \in \Sigma$ is such that $\mu(B)=\mu(B \backslash A)=\mu(A) / 2$.

This yields that equality (2) does not hold, and the proof is finished.

Remark 1. If $\mu(\Omega)=\infty$, then conditions $u_{\infty}(\varphi)<\infty$ and $\varphi\left(u_{\infty}(\varphi)\right)=0$ from Theorem 2 and $u_{0}(\varphi)>0, u_{\infty}(\varphi)<\infty$ and $u_{0}(\varphi)=u_{\infty}(\varphi)$ from Theorem 3 are equivalent. This means that in the case of nonatomic infinite measure space the Orlicz space with the Luxemburg-Nakano norm is an AM-space if and only if the Orlicz space with the Amemiya norm is also an AM-space, and this is equivalent to the fact that $\varphi(u)=0$ for $0 \leqq u \leqq u_{0}$ and $\varphi(u)=\infty$ for $u>u_{0}$ for some $u_{0}>0$. The difference can appear only in the case when $\mu(\Omega)<\infty$.

Example 1. For a fixed $c>0$ and $p \geqq 1$ let

$$
\varphi(u)= \begin{cases}u^{p} & \text { for } 0 \leqq u \leqq c \\ \infty & \text { for } \quad u>c\end{cases}
$$

Then $u_{0}(\varphi)=0, u_{\infty}(\varphi)=c$ and $L_{\varphi}([a, b])=L_{p}([a, b]) \cap L_{\infty}([a, b])=L_{\infty}([a, b])$ with

$$
\|x\|_{\varphi}=\max \left\{\|x\|_{p}, c^{-1}\|x\|_{\infty}\right\} \quad \text { and } \quad\|x\|_{\varphi}^{A}=\|x\|_{p}+c^{-1}\|x\|_{\infty} .
$$

Note that if $(b-a) c^{p} \leqq 1$, then $\|x\|_{\varphi}=c^{-1}\|x\|_{\infty}$.

3. Orlicz sequence spaces which are AM-spaces. In Orlicz sequence spaces the case of Luxemburg-Nakano norm is easy again.

Theorem 4. An Orlicz sequence space $l_{\varphi}$ with the Luxemburg-Nakano norm is an AM-space if and only if

$$
u_{0}(\varphi)>0, u_{\infty}(\varphi)<\infty \quad \text { and } \quad u_{0}(\varphi)=u_{\infty}(\varphi)
$$

Proof. Assume that (4) does not hold, i.e., either $u_{0}(\varphi)=0$ or $u_{0}(\varphi) \neq u_{\infty}(\varphi)$. If $u_{0}(\varphi)=0$, then $l_{\varphi}$ is strictly monotone (see [6]), so it cannot be an AM-space. If $u_{0}(\varphi) \neq u_{\infty}(\varphi)$, then there exists $u>0$ such that $0<\varphi(u)<\infty$. Then we can find $v \in[0, u]$ and a natural number $n$ such that $n \varphi(v)=1$. Define

$$
x=(v, \ldots, v, 0,0, \ldots), y=(0, \ldots, 0, v, \ldots, v, 0,0, \ldots) .
$$

We have $\quad I_{\varphi}(x)=I_{\varphi}(y)=n \varphi(v)=1 \quad$ and $\quad$ so $\quad\|x\|_{\varphi}=\|y\|_{\varphi}=1 . \quad$ Moreover, $I_{\varphi}(x+y)=2 n \varphi(v)=2$. Thus $\|x+y\|_{\varphi}>1=\max \left(\|x\|_{\varphi},\|y\|_{\varphi}\right)$, which means that $l_{\varphi}$ with the Luxemburg-Nakano norm is not an AM-space.

The case of Orlicz sequence space with the Orlicz norm contains more possibilities. Denote by $\varphi_{+}^{\prime}$ the right derivative of $\varphi$.

Theorem 5. The following are equivalent:

(i) An Orlicz sequence space $l_{\varphi}$ with the Orlicz norm is an AM-space.

(ii) $l_{\varphi}=l_{\infty}$ and there is a constant $c>0$ such that $\|x\|_{\varphi}^{0}=c\|x\|_{\infty}$ for any $x \in l_{\varphi}$. 
(iii) $u_{0}(\varphi) \varphi_{+}^{\prime}\left(u_{0}(\varphi)\right) \geqq 1$.

(iv) $\varphi^{*}$ is linear on the interval $\left[0, u_{1}\right]$, where $\varphi^{*}\left(u_{1}\right)=1$.

(v) $l_{\varphi^{*}}=l_{1}$ and there is a constant $k>0$ such that $\|x\|_{\varphi^{*}}=k\|x\|_{1}$ for any $x \in l_{\varphi^{*}}$.

Proof. (i) $\Rightarrow$ (ii). Note that (i) implies that $u_{0}(\varphi)>0$, because conversely $l_{\varphi}$ is strictly monotone (see [3]), so it cannot be an AM-space. This also follows by the fact that if $l_{\varphi}$ is an AM-space, then by virtue of the Fatou property of $l_{\varphi}$, we have $\chi_{N} \in l_{\varphi}$, i.e., $l_{\infty} \subset l_{\varphi}$ but this yields that $u_{0}(\varphi)>0$. Indeed, if $\left(l_{\varphi},\|\cdot\|_{\varphi}^{0}\right)$ is an AM-space, then for any $k, n \in N, n>k$, we have

$$
\begin{aligned}
\left\|\sum_{i=k}^{n} e_{i}\right\|_{\varphi}^{0} & =\left\|\max \left(e_{k}, e_{k+1}, \ldots, e_{n}\right)\right\|_{\varphi}^{0} \\
& =\max \left(\left\|e_{k}\right\|_{\varphi}^{0},\left\|e_{k+1}\right\|_{\varphi}^{0}, \ldots,\left\|e_{n}\right\|_{\varphi}^{0}\right)=c .
\end{aligned}
$$
Therefore by the Fatou property of $\|\cdot\|_{\varphi}^{0}$, we get that $\sum_{i=k}^{\infty} e_{i} \in l_{\varphi}$ and $\left\|\sum_{i=k}^{\infty} e_{i}\right\|_{\varphi}^{0}=c$ for any
$k \in N$. Hence we can easily get that

$$
\left\|\chi_{A}\right\|_{\varphi}^{0}=\left\|\sum_{i \in A} e_{i}\right\|_{\varphi}^{0}=c \text { for any } A \subset N, A \neq \emptyset .
$$

Now, we will show that $l_{\varphi} \subset l_{\infty}$. Let $x \in l_{\varphi}$. If $x \notin l_{\infty}$, then for any $k \in N$ there exist $n_{k} \in N$ such that $\left|x_{n_{k}}\right|>k$. Therefore, for each $k \in N$,

$$
\|x\|_{\varphi}^{0} \geqq\left\|\left|x_{n_{k}}\right| e_{n_{k}}\right\|_{\varphi}^{0}>k\left\|e_{n_{k}}\right\|_{\varphi}^{0}=k c .
$$

By the arbitrariness of $k \in N$ we get $\|x\|_{\varphi}^{0}=\infty$, a contradiction. Thus $l_{\varphi} \subset l_{\infty}$. We even will show that $l_{\varphi}=l_{\infty}$ and $\|x\|_{\varphi}^{0}=c\|x\|_{\infty}$. For any $x \in l_{\varphi}, x \neq 0$, we have $\|x /\| x\left\|_{\infty}\right\|_{\varphi}^{0} \leqq\left\|\chi_{\text {supp } x}\right\|_{\varphi}^{0}=c$, i.e. $\|x\|_{\varphi}^{0} \leqq c\|x\|_{\infty}$.

On the other hand, take any $\lambda \in(0,1)$ and any $x \in l_{\varphi}, x \neq 0$. There exists $n \in N$ such that $\left|x_{n}\right|>\lambda\|x\|_{\infty}$, whence

$$
\|x /\| x\left\|_{\infty}\right\|_{\varphi}^{0} \geqq\left\|\lambda e_{n}\right\|_{\varphi}^{0}=\lambda\left\|e_{n}\right\|_{\varphi}^{0}=\lambda c,
$$

and by arbitrariness of $\lambda \in(0,1),\|x\|_{\varphi}^{0} \geqq c\|x\|_{\infty}$. Thus $\|x\|_{\varphi}^{0}=c\|x\|_{\infty}$.

(ii) $\Rightarrow$ (i). This implication is obvious.

(ii) $\Leftrightarrow(\mathrm{v})$. Since $l_{\infty}, l_{1}$ and $\left(l_{\varphi},\|\cdot\|_{\varphi}^{0}\right),\left(l_{\varphi^{*}},\|\cdot\|_{\varphi^{*}}\right)$ are two couples of mutually dual spaces in the sense of Köthe ( for the Köthe duality see e.g. [7] ), we deduce that (ii) is equivalent to $(\mathrm{v})$.

(iii) $\Rightarrow$ (iv). Let q denote the generalized inverse function of $\varphi_{+}^{\prime}$, i.e.,

$$
q(t)=\sup \left\{s>0: \varphi_{+}^{\prime}(s)<t\right\} \text { with } \operatorname{supp} \emptyset=0 .
$$

Then we have in our case $q(t)=u_{0}(\varphi)$ for $t \in\left[0, \varphi_{+}^{\prime}\left(u_{0}(\varphi)\right)\right]$. Therefore $\varphi^{*}(u)=\int_{0}^{u} q(t) d t$ is linear on the interval $\left[0, \varphi_{+}^{\prime},\left(u_{0}(\varphi)\right)\right]$ and

$$
\varphi^{*}\left(\varphi_{+}^{\prime}\left(u_{0}(\varphi)\right)\right)=u_{0}(\varphi) \varphi_{+}^{\prime}\left(u_{0}(\varphi)\right) \geqq 1 .
$$

Thus (iv) holds with $u_{1} \leqq \varphi_{+}^{\prime}\left(u_{0}(\varphi)\right)$.

The implication (iv) $\Rightarrow$ (iii) can be proved analogously. 
(iv) $\Rightarrow(\mathrm{v})$. Assumption (iv) gives that $\varphi^{*}(u)=u / u_{1}$ for $u \in\left[0, u_{1}\right]$. We will show that if $x \in l_{\varphi^{*}}, x \neq 0$, then $\|x\|_{\varphi^{*}}=u_{1}^{-1}\|x\|_{1}$.

We have $I_{\varphi^{*}}\left(x /\|x\|_{\varphi^{*}}\right) \leqq 1$. This implies $\varphi^{*}\left(\left|x_{n}\right| /\|x\|_{\varphi^{*}}\right) \leqq 1$ for all $n \in N$, and so $\left|x_{n}\right| /\|x\|_{\varphi^{*}} \leqq u_{1}$, which gives $\varphi^{*}\left(\left|x_{n}\right| /\|x\|_{\varphi^{*}}\right)=\left|x_{n}\right| /\left(\|x\|_{\varphi^{*}} u_{1}\right)$. By summation we obtain

$$
\begin{aligned}
1 & \geqq I_{\varphi^{*}}\left(x /\|x\|_{\varphi^{*}}\right)=\sum_{n=1}^{\infty} \varphi^{*}\left(\left|x_{n}\right| /\|x\|_{\varphi^{*}}\right) \\
& =\sum_{n=1}^{\infty}\left|x_{n}\right| /\left(\|x\|_{\varphi^{*}} u_{1}\right)=\|x\|_{1} /\left(\|x\|_{\varphi^{*}} u_{1}\right),
\end{aligned}
$$

i.e. $\|x\|_{\varphi^{*}} \geqq\|x\|_{1} / u_{1}$.

On the other hand,

$$
I_{\varphi^{*}}\left(x u_{1} /\|x\|_{1}\right)=\sum_{n=1}^{\infty} \varphi^{*}\left(\left|x_{n}\right| u_{1} /\|x\|_{1}\right)=\sum_{n=1}^{\infty}\left|x_{n}\right| /\|x\|_{1}=1,
$$

and so $\left\|x u_{1} /\right\| x\left\|_{1}\right\|_{\varphi^{*}} \leqq 1$, which gives $\|x\|_{\varphi^{*}} \leqq\|x\|_{1} / u_{1}$. Therefore,

$$
\|x\|_{\varphi^{*}}=\|x\|_{1} / u_{1} .
$$

(v) $\Rightarrow$ (iv). Note first that condition (v) implies that there is $u_{1}>0$ such that $\varphi^{*}\left(u_{1}\right)=1$. Denote $\Psi=\varphi^{*}$ and assume for the contrary that $\Psi\left(u_{\infty}(\Psi)\right)<1$.

Defining $x=\left(u_{\infty}(\Psi), 0,0, \ldots\right)$, we get $I_{\Psi}(x)=\Psi\left(u_{\infty}(\Psi)\right)<1$ and for any $\lambda \in(0,1)$, we have $I \Psi(x / \lambda)=\Psi\left(u_{\infty}(\Psi) / \lambda\right)=\infty$, whence $\|x\|_{\Psi}=1$. Let $b>0$ be such that $\Psi\left(u_{\infty}(\Psi)\right)+\Psi(b) \leqq 1$ and define $\left.y=\left(u_{\infty}(\Psi)\right), b, 0,0, \ldots\right)$. Then $\|y\|_{\Psi}=1$ and $\|x\|_{1}=u_{\infty}(\Psi),\|y\|_{1}=u_{\infty}(\Psi)+b>u_{\infty}(\Psi)$, and so $l_{\Psi}$ and $l_{1}$ cannot be isometric under the isometry $\lambda$ Id for some $\lambda>0$. So, we have proved that condition (v) implies that $\Psi\left(u_{\infty}(\Psi)\right) \geqq 1$. Assume without loss of generality that $\Psi(1)=1$ (since we can take a new function $\phi(u)=\Psi\left(u u_{1}\right)$ for which $\phi(1)=1$ and $\left.\|\cdot\|_{\phi}=u_{1}\|\cdot\| \Psi\right)$. Then we need to prove that $\Psi$ is linear on the interval $[0,1]$. Assume for the contrary that $\Psi$ is not linear on the interval $[0,1]$. Then $\Psi(1 / 2)<\Psi(1) / 2=1 / 2$. Therefore, defining $x=(1 / 2,1 / 2,0,0, \ldots)$, we get $\|x\|_{1}=1$ but $I \Psi(x)=2 \Psi(1 / 2)<1$, whence it follows that $\|x\|_{\Psi}<1$. This shows that $l_{\Psi}$ is not then isometric to $l_{1}$ under the identity mapping. It is obvious that if $\Psi(1)=1$ and $\Psi$ is linear on $[0,1]$, then $\|x\|_{\psi}=\|x\|_{1}$ for any $x \in l \Psi$. We can prove in the same way that $\|x\|_{\Psi}=k\|x\|_{1}$ for any $x \in l_{\Psi}$ if and only if $\Psi(1 / k)=1$ and $\Psi$ is linear on the interval $[0,1 / k]$.

Example 2. For a fixed $c>1$ let $\varphi(u)=0$ for $0 \leqq u \leqq 1 / c, \varphi(u)=c u-1$ for $1 / c \leqq u \leqq 1$ and $\varphi(u)=\infty$ for $u>1$. Then $l_{\varphi}=l_{\infty}$ with $\|x\|_{\varphi}^{A}=c\|x\|_{\infty}$. On the other hand, for any nonempty finite subset $A$ of $N$ we have $\left\|\chi_{A}\right\|_{\varphi}=\max \{1, c|A| /(1+|A|)\}$, which shows that $l_{\varphi}$ with the Luxemburg-Nakano norm is not an AM-space.

Remark 2. Let us define for any Orlicz function $\varphi$, the subspace $E_{\varphi}$ of $L_{\varphi}$ as the closure of the set of simple functions in the space $L_{\varphi}$. In the sequence case let us define $h_{\varphi}$ to be the closure in $l_{\varphi}$ of the space of all sequences with finite number of coordinates different from zero. Consider the spaces $E_{\varphi}$ and $h_{\varphi}$ with the Luxemburg-Nakano and the Amemiya norm induced from $L_{\varphi}$ (resp. $l_{\varphi}$ ). These norms are order continuous in $E_{\varphi}$ and $h_{\varphi}$ but they do not have the Fatou property. Sine $l_{\infty}$ is not order continuous the equalities $E_{\varphi}=L_{\infty}$ and 
$l_{\varphi}=l_{\infty}$ are impossible. Note that $l_{\infty}=l_{\varphi}$ and $c_{0}=h_{\varphi}$ isometrically when $\varphi(u)=0$ for $0 \leqq u \leqq 1$ and $\varphi(u)=\infty$ for $u \leqq 1$. It is obvious that both $l_{\infty}$ and $c_{0}$ are AM-spaces. So, it is natural to ask when $E_{\varphi}$ and $h_{\varphi}$ are AM-spaces. Note that if we replace equalities $E_{\varphi}=L_{\infty}$ and $l_{\varphi}=l_{\infty}$ by the inclusions $E_{\varphi} \subset L_{\infty}$ and $l_{\varphi} \subset l_{\infty}$, respectively, then all the theorems remain valid for $E_{\varphi}$ and $h_{\varphi}$ in place of $L_{\varphi}$ and $l_{\varphi}$, respectively. The sufficiency is obvious and in the necessity part we always constructed simple functions or sequences with finite number of coordinates different from zero, which were in fact in $E_{\varphi}$ or $h_{\varphi}$, respectively.

\section{References}

[1] K. BARon and H. HUdZiK, Orlicz spaces which are $L^{p}$-spaces. Aequationes Math. 48, 254-261 (1994).

[2] H. HudziK, Orlicz spaces of essentially bounded functions and Banach-Orlicz algebras. Arch. Math. 44, 535-538 (1985).

[3] H. HudzIK and W. KURC, Monotonicity properties of Musielak-Orlicz spaces and best approximation in Banach lattices. J. Approx. Theory, to appear.

[4] H. HudziK and L. Maligranda, Amemiya norm and Orlicz norm are equal, to appear.

[5] M. A. Krasnosel'skit and J. B. RutickiI, Convex Functions and Orlicz Spaces. Groningen 1961.

[6] W. KurC, Strictly and uniformly monotone Musielak-Orlicz spaces and applications to best approximation. J. Approx. Theory 69, 173-187 (1992).

[7] J. Lindenstrauss and L. Tzafriri, Classical Banach Spaces II. Function Spaces. BerlinHeidelberg-New York 1979.

[8] L. MaligRanda, Orlicz Spaces and Interpolation. Seminars in Math. 5, Campinas 1989.

[9] J. MusielaK, Orlicz Spaces and Modular Spaces. LNM 1034. Berlin-Heidelberg-New York 1983.

[10] M. M. RAO and Z. D. Ren, Theory of Orlicz Spaces. Pure Appl. Math. 146, New York 1991.

Eingegangen am 2. 7. 1996*)

Anschriften der Autoren:

C. E. Finol

Departamento de Matemáticas

Facultad de Ciencias

Universidad Central de Venezuela

Apartado 20513

Caracas 1020-A

Venezuela
H. Hudzik

Faculty of Mathematics and Computer Science

Adam Mickiewicz University

Matejki 48/49

60-769 Poznań

Poland
L. Maligranda

Department of Mathematics

Luleå University

S-971 87 Luleå

Sweden

*) Die vorliegende Fassung ging am 14. 1. 1997 ein. 